\title{
Complexation of amphotericin B and curcumin with serum albumins: solubility and effect on erythrocyte membrane damage
}

This article was published in the following Dove Press journal:

Journal of Experimental Pharmacology

31 December 2010

Number of times this article has been viewed

\author{
Avinash K Kudva' \\ MN Manoj² \\ Bale M Swamy' \\ Candadai S Ramadoss ${ }^{3}$ \\ 'Department of Biochemistry, \\ Karnatak University, Dharwad, India; \\ ${ }^{2}$ Bigtec Labs, SID Entrepreneurship \\ Centre, ${ }^{3}$ Unichem Laboratories Ltd, \\ Biosciences R\&D Centre, IISc campus, \\ Bangalore, India
}

\begin{abstract}
Amphotericin and curcumin are known to form complexes with albumins individually. In-silico analysis shows that amphotericin B and curcumin have separate binding regions on human serum albumin and bovine serum albumin. The complex formed with albumin in the presence of both amphotericin and curcumin is water soluble, and it retains antifungal activity. Interestingly, it was found that the presence of curcumin in the complex significantly delayed the red cell lysis by amphotericin B, indicating the possibility of moderating the toxic side effects of the drug using curcumin. Furthermore, since the presumed ternary complex is stable and water soluble, its potential use in the treatment of visceral leishmaniasis (kala azar) and systemic fungal infections needs to be evaluated.
\end{abstract}

Keywords: hemolytic activity, antifungal activity

\section{Introduction}

Fungal infections, both community acquired and nosocomial represent a major challenge, particularly in immunocompromised patients, as the organisms tend to be resistant to common drugs. Amphotericin B (AmB), a potent polyene is one of the drugs used in therapy for patients with deep-seated fungal infections caused by acquired immunodeficiencies and transplantations. ${ }^{1}$ Though it remains a potent antifungal drug, its use is hampered by its low solubility in water and toxic side effects. ${ }^{2}$ To circumvent these side effects, various drug formulations such as liposomes, deoxycholate salts, and nanospheres have been reported and used for treatment. ${ }^{2}$ Some of these formulations are less toxic than others, but each has its own limitations. To circumvent these limitations, the interaction of AmB with serum albumins has been studied by a few groups, notably by Butler et al and Romanini et al. ${ }^{3,4}$

Serum albumins are known for their ability to bind a variety of ligands. This property helps to render a water-insoluble substance soluble and transportable. ${ }^{6}$ Their lack of immunogenicity and toxicity coupled with abundant availability makes them ideal candidates for drug delivery. Human serum albumin (HSA) is used as an excipient in some drugs and is also used for treating shock, burns, and acute respiratory distress, and in hemodialysis. ${ }^{5,6}$ Bovine serum albumin (BSA) was also investigated as it is structurally homologous to HSA. ${ }^{7}$ Considering the significant advantages of serum albumin as a drug delivery system, we resorted to in-silico analysis to identify the putative binding regions of $\mathrm{AmB}$ to serum albumin.
Correspondence: Bale M Swamy Department of Biochemistry, Karnatak University, Dharwad-580003,

Karnataka, India

$\mathrm{Tel}+9 \mid 8362771973$

Fax +91 8362747884

Email sawmy_bm@yahoo.co.in
Journal of Experimental Pharmacology 201 I:3 I-6

(C) 20II Kudva et al, publisher and licensee Dove Medical Press Ltd. This is an Open Access article which permits unrestricted noncommercial use, provided the original work is properly cited. 


\section{Binding of AmB with albumins in-silico study}

Earlier reports by Romanini et al suggest that in HSA the residues Lysine 199 and 525 participate in AmB-HSA interaction with binding energies around $10^{5} \mathrm{M}^{-1}{ }^{4}$ For our analysis, AutoDock 3.0 suite developed by Scripps Research Institute was used to compute and determine the region of AmB binding on serum albumins. ${ }^{8}$ Our docking results indicate that both human and bovine serum albumins have only one favorable binding site each for AmB. AmB binds to HSA away from the central cleft of the molecule and interacts with six amino acids (at $3 \AA$ vicinity) in domain IIIA. These include, two Glu $(392,396)$ and two $\operatorname{Gln}(518,400)$ residues and one residue each of Arg424 and Ser431 (Figure 1). On BSA, AmB binds in the cleft region, interacting with seven amino acids, of which two are Lys $(138,455)$ and one residue each of Asp135, Glu423, Gln427, Arg451, Thr542 (Figure 2).

\section{Spectral data of AmB-albumin complex}

The AmB complexes were studied by monitoring spectral changes with UV-visible spectroscopy. The spectra of both BSA and HSA complexed with AmB had some characteristic perturbations especially at around $335-340 \mathrm{~nm}$ (Figures $3 \mathrm{~A}$ and $4 \mathrm{~A}$ ). AmB in phosphate-buffered saline

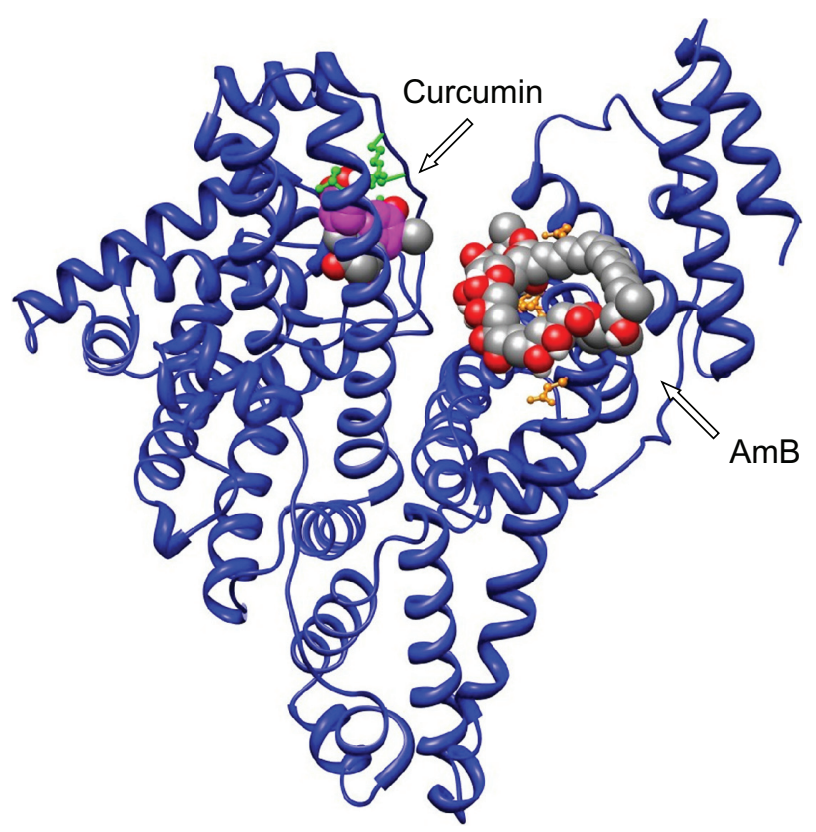

Figure I In silico analysis: binding of $A m B$ and curcumin to human serum albumin. Curcumin interacts with domain IB, whereas AmB interacts with domain IIIA comprising two Glu $(392,396)$, two $G \ln (518,400)$ residues, and one each of $\operatorname{Arg} 424$ and Ser43I residues.

Abbreviation: AmB, amphotericin B.

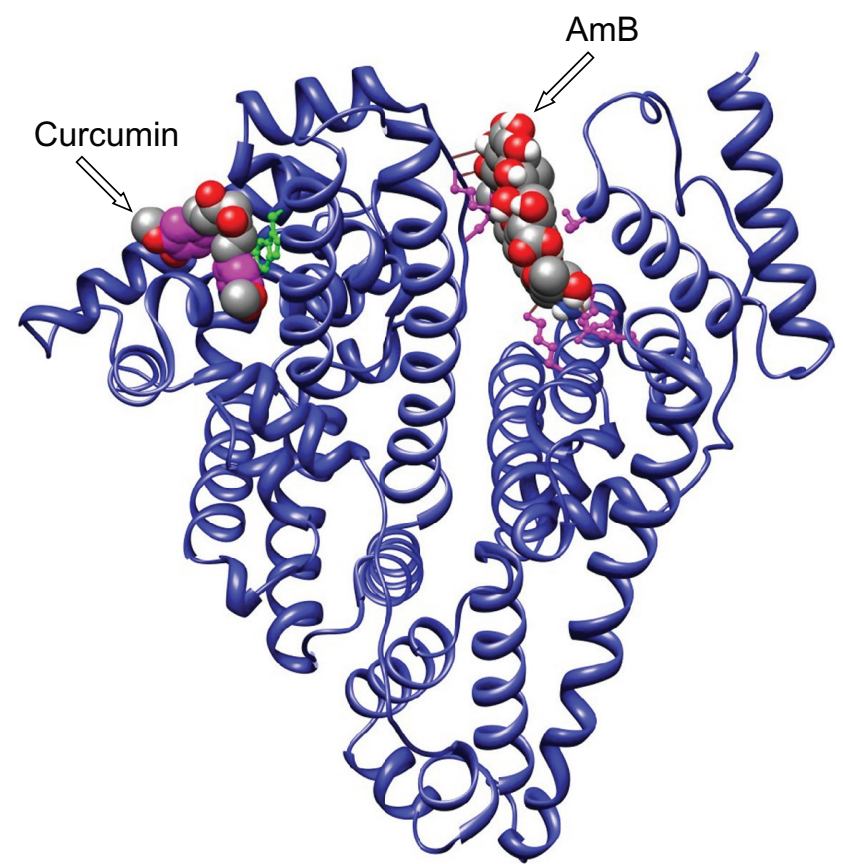

Figure 2 In silico analysis: binding of $A m B$ and curcumin to bovine serum albumin. Curcumin binds to the vicinity of Trp I58, whereas AmB binds in the cleft, interacting with amino acid residues Lys 138, Lys455, Asp I35, Glu423, Gln427, Arg45I, Thr542. Abbreviation: AmB, amphotericin B.

with $1 \%$ dimethyl sulfoxide (DMSO) displayed a characteristic peak at $340 \mathrm{~nm}$, while the complex showed a spectral blue-shift of about $5 \mathrm{~nm}$. In addition, a hyperchromic peak was observed, which may be attributed to the change in the environment of the molecule. The increasing absorbance at $335 \mathrm{~nm}$ is similar to the changes of the AmB spectrum displayed in the aggregated state in water. Romanini et al observed and postulated that this hyperchromicity is due to the "self perturbation" of the AmB dipole during dimer formation and is caused by dipolar forces involving polar groups present in the AmB molecule. ${ }^{4}$ Such prior observations agree with the notion that the AmB monomer interacts with the polar groups present on the bound serum albumin.

\section{Bioactivity of AmB-albumin complex}

The antifungal activities of the AmB-albumin (HSA and BSA) complexes were tested by broth dilution against Candida albicans and C. glabrata. Results from these experiments suggest that the complex retains antifungal activity that is equivalent to the drug alone (Table 1).

Since AmB is known to cause lysis of erythrocytes, the complexes were also tested for hemolytic activity on human erythrocytes. ${ }^{9}$ The results showed that the prepared complexes have only a marginal protective effect against membrane damage (Figure 6, samples E and F). 

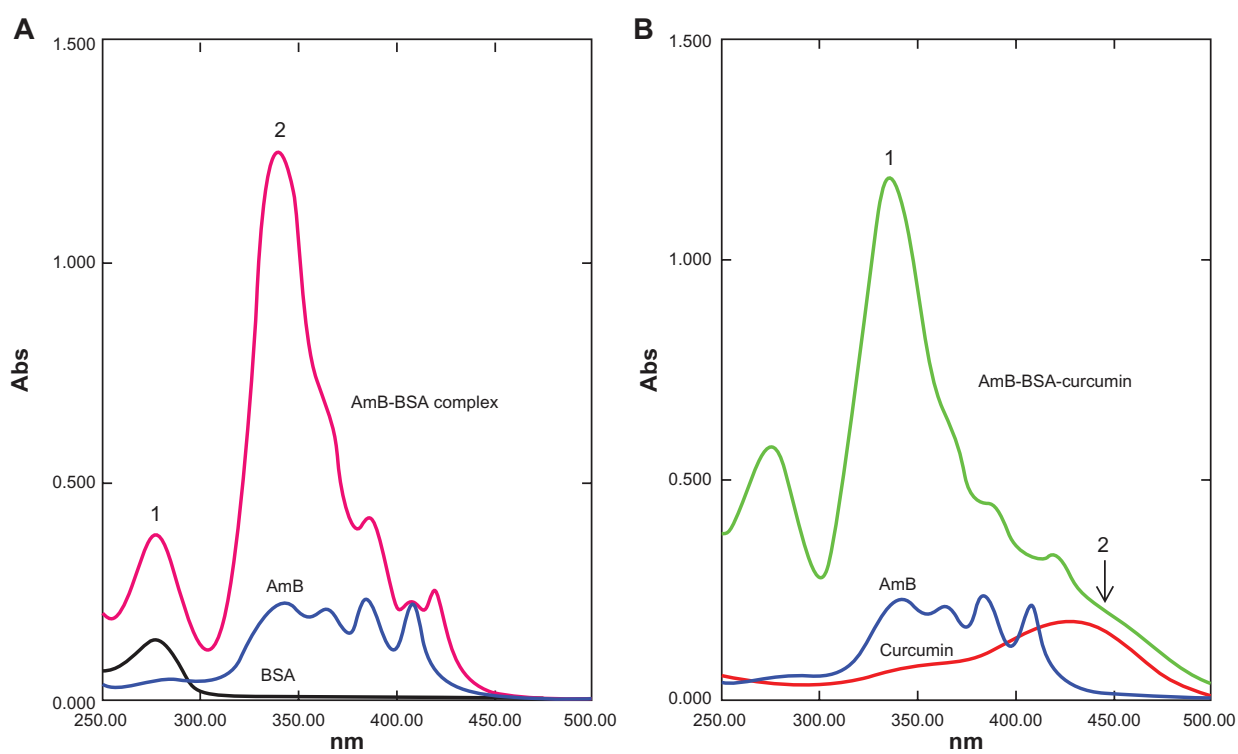

Figure 3 Ultraviolet-visible spectral analysis of AmB, curcumin, BSA, AmB-BSA complex, and AmB-BSA-curcumin complex. A) Spectra of the AmB-BSA complex showing a blue-shift from $340 \mathrm{~nm}$ to $335 \mathrm{~nm}$ compared with free AmB. A hyperchromic peak (peak 2) at $335 \mathrm{~nm}$ was observed. ${ }^{7}$ Peak I represents the absorbance peak of albumin. B) Spectra of the AmB-BSA-curcumin complex showing a hyperchromic peak at $335 \mathrm{~nm}$ (peak I). In addition, there is a broad shoulder around $450 \mathrm{~nm}$ indicating the presence of curcumin (peak 2).

Abbreviations: AmB, amphotericin B; BSA, bovine serum albumin.

Several published reports show that AmB has a permeabilizing effect on red blood cell membrane. ${ }^{9}$ Brajtburg et al have reported that the auto-oxidation of the polyene drug releasing reactive oxygen species leads to these anticellular effects. ${ }^{10}$ Hence, we thought that the addition of a free radical scavenger or an antioxidant in the above albumin-AmB complex would help to lessen the hemolytic activity of the drug.

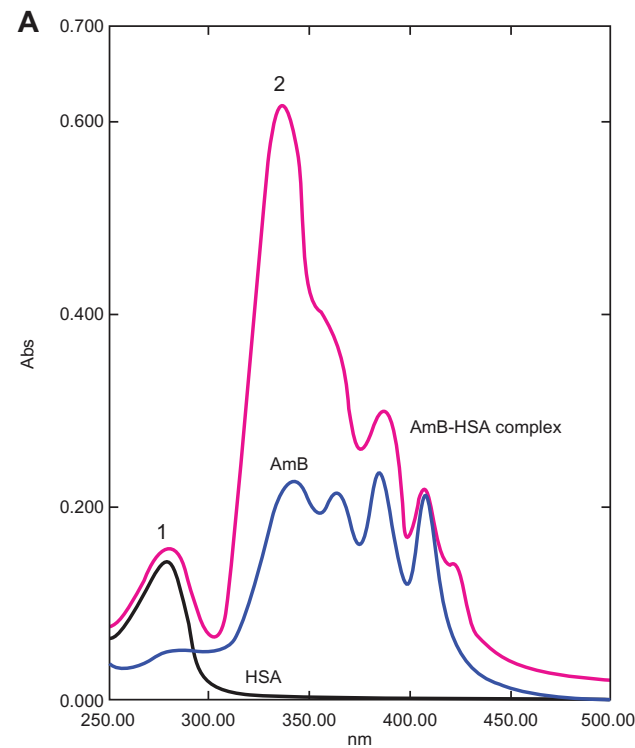

\section{Binding of curcumin to albumins: In-silico study and bioactivity}

Curcumin, a yellow pigment isolated from the rhizomes of Curcuma longa has been reported to possess antioxidant activity and known to exhibit various pharmacological effects including antimicrobial and anticancer properties. ${ }^{11}$ Coincidently, curcumin too is insoluble in aqueous medium

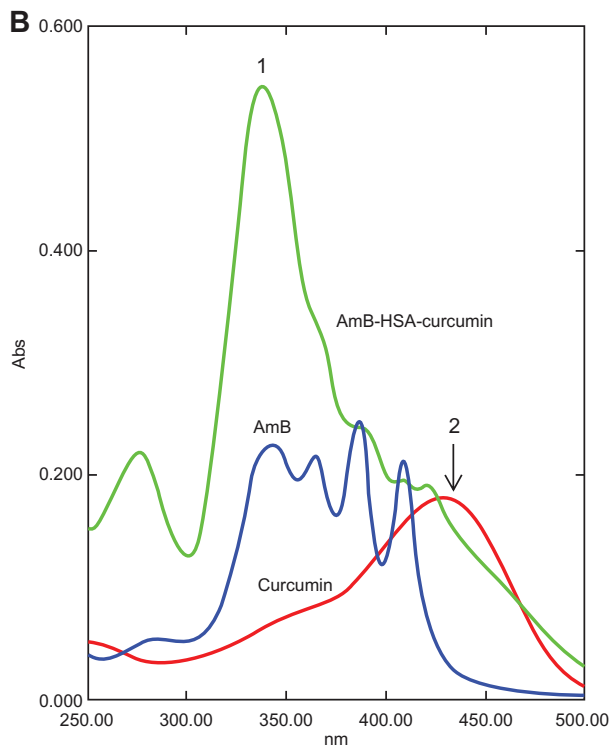

Figure 4 Ultraviolet-visible spectral analysis of AmB, curcumin, AmB-HSA complex, and AmB-HSA-curcumin complex. A) The AmB-HSA complex showed a blue-shift from $340 \mathrm{~nm}$ to $335 \mathrm{~nm}$ compared with free AmB (peak I). A hyperchromic peak (peak 2) at $335 \mathrm{~nm}$ was also observed. ${ }^{7}$ Peak I represents the absorbance peak of albumin. B) Spectra of the AmB-HSA-curcumin complex showing a hyperchromic peak at $335 \mathrm{~nm}$ (peak I). In addition, there is a broad shoulder around $450 \mathrm{~nm}$ indicating the presence of curcumin (peak 2).

Abbreviations: AmB, amphotericin B; HSA, human serum albumin. 
Table I In vitro antifungal activity (Broth dilution method) of free and complexed amphotericin B carried out according to NCCLS guidelines ${ }^{20}$

\begin{tabular}{|c|c|c|c|}
\hline \multirow[t]{2}{*}{ Preparation } & \multicolumn{3}{|c|}{ Fungal strain/MIC $(\mu \mathrm{g} / \mathrm{mL})^{\mathrm{a}}$} \\
\hline & $\begin{array}{l}\text { Candida albicans } \\
\text { (NCIM 3466) }\end{array}$ & $\begin{array}{l}\text { Candida glabrata } \\
\text { (NCIM 3236) }\end{array}$ & $\begin{array}{l}\text { Candida krusei } \\
\text { (NCIM 35I8) }\end{array}$ \\
\hline Free amphotericin $\mathrm{B}^{\mathrm{b}}$ & 0.2 & 0.3 & 0.35 \\
\hline AmB-BSA complex & 0.2 & 0.3 & 0.3 \\
\hline AmB-HSA complex & 0.2 & 0.3 & 0.3 \\
\hline AmB-BSA-curcumin complex & 0.2 & 0.3 & 0.3 \\
\hline AmB-HSA-curcumin complex & 0.2 & 0.3 & 0.3 \\
\hline
\end{tabular}

Notes: MIC values show that all the water-soluble complexes retain antifungal activities equivalent to the free drug; ${ }^{\text {a }} \mathrm{Values}$ are representative of two different experiments performed in duplicate; ${ }^{b}$ Free amphotericin was dissolved in DMSO and serially diluted with saline. Final test concentrations for the free AmB assays contained $<5 \%$ DMSO.

Abbreviations: AmB, amphotericin B; BSA, bovine serum albumin; DMSO, dimethyl sulfoxide; HSA, human serum albumin; MIC, minimum inhibitory concentration; NCCLS, National Committee for Clinical Laboratory Standards; NCIM, National Collection of Industrial Microorganisms.

and is poorly absorbed by the body. ${ }^{12}$ Various formulations like polymeric nanoparticles and liposomes have been prepared to increase the bioavailability. ${ }^{13,14}$ Incidentally, Tsao et al reported that the presence of curcumin enhanced the inhibitory activity of AmB against Candida spp. ${ }^{15}$ This report, combined with the known antioxidant property, prompted us to investigate the use of curcumin along with AmB.

There are a few reports on the complexation of curcumin with serum albumin. Barik et al have reported the formation of BSA-curcumin complex with binding constants in the order of $10^{4}-10^{5} \mathrm{M}^{-1} .{ }^{16}$ The same group has also studied the formation of HSA-curcumin complex and reported that the site of curcumin binding to HSA is likely to be at domain $I I A$, with binding constant estimated to be around $7.9 \times 10^{5} \mathrm{M}^{-1} .{ }^{17}$ The latest report from Bourassa et al on curcumin binding to BSA indicate the number of bound polyphenols (n) to be 1.0 and the binding constant to be $3.33( \pm 0.8) \times 10^{4} \mathrm{M}^{-1} .{ }^{7}$ The docking simulations carried out by us indicate the presence of a single binding site on HSA, located away from the Trp214, on domain IB (Figure 1). With BSA, curcumin is more likely to bind in the vicinity of Trp158 (Figure 2). The proposed complex of curcumin with HSA/BSA was prepared, and spectral properties determined (Figure 5). The curcumin-albumin complex showed no spectral changes and was essentially similar to that of curcumin. In addition the curcumin-albumin complex showed no intrinsic antifungal activity (data not shown) and was found to be nonhemolytic in nature (Figure 6, sample B).

\section{AmB-Albumin-Curcumin complex: spectral data and bioactivity}

Since the binding site of curcumin was located in a different domain of albumins (Figures 2 and 3), there was a distinct possibility of formation of ternary complex involving albumins, AmB, and curcumin. We therefore prepared an albumin, AmB, and curcumin complex. The prepared complex was water soluble ( $\sim 10 \mathrm{mg}$ of protein $/ \mathrm{mL}$ of water). The spectrum of such a complex is shown in Figures 3B and $4 \mathrm{~B}$. The spectral properties indicate the presence of hyperchromic peak at $335 \mathrm{~nm}$ similar to AmB-albumin complex with a broad shoulder around $450 \mathrm{~nm}$ representative of curcumin.

Testing of a mixture of free AmB and curcumin in the in vitro red cell lysis assay shows that the lysis induced by the antifungal agent is delayed (Figure 6, sample D).

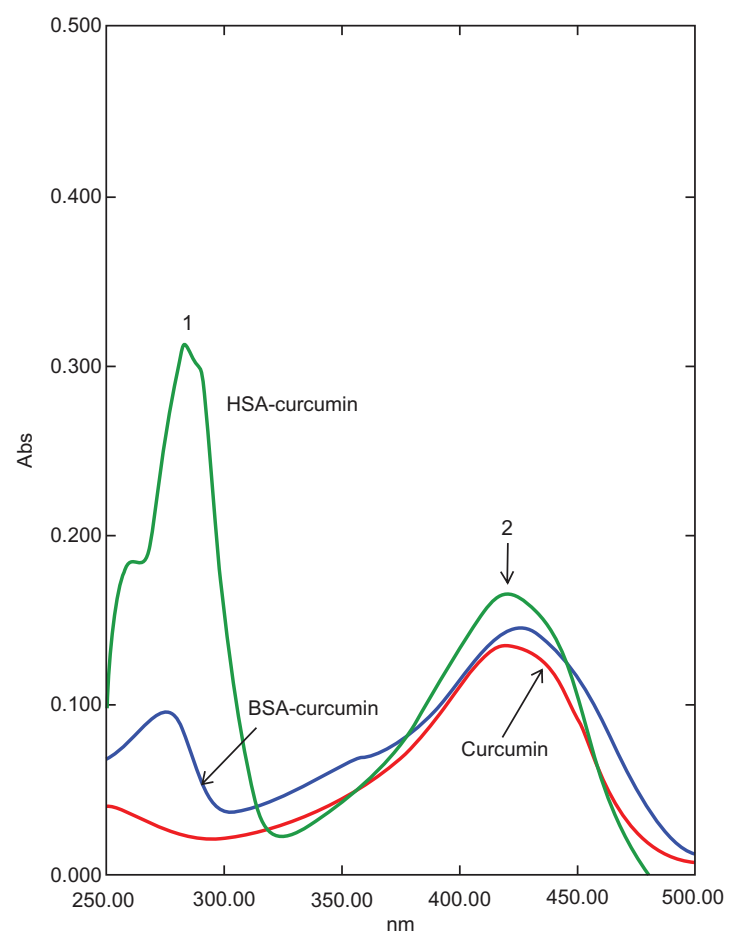

Figure 5 Ultraviolet-visible spectral analysis of curcumin, curcumin-HSA complex, and curcumin-BSA complex. The curcumin-albumin complexes show similar spectra properties as that of curcumin alone (peak 2). Peak I represents the absorbance peak of albumin.

Abbreviations: AmB, amphotericin B; BSA, bovine serum albumin; HSA, human serum albumin. 


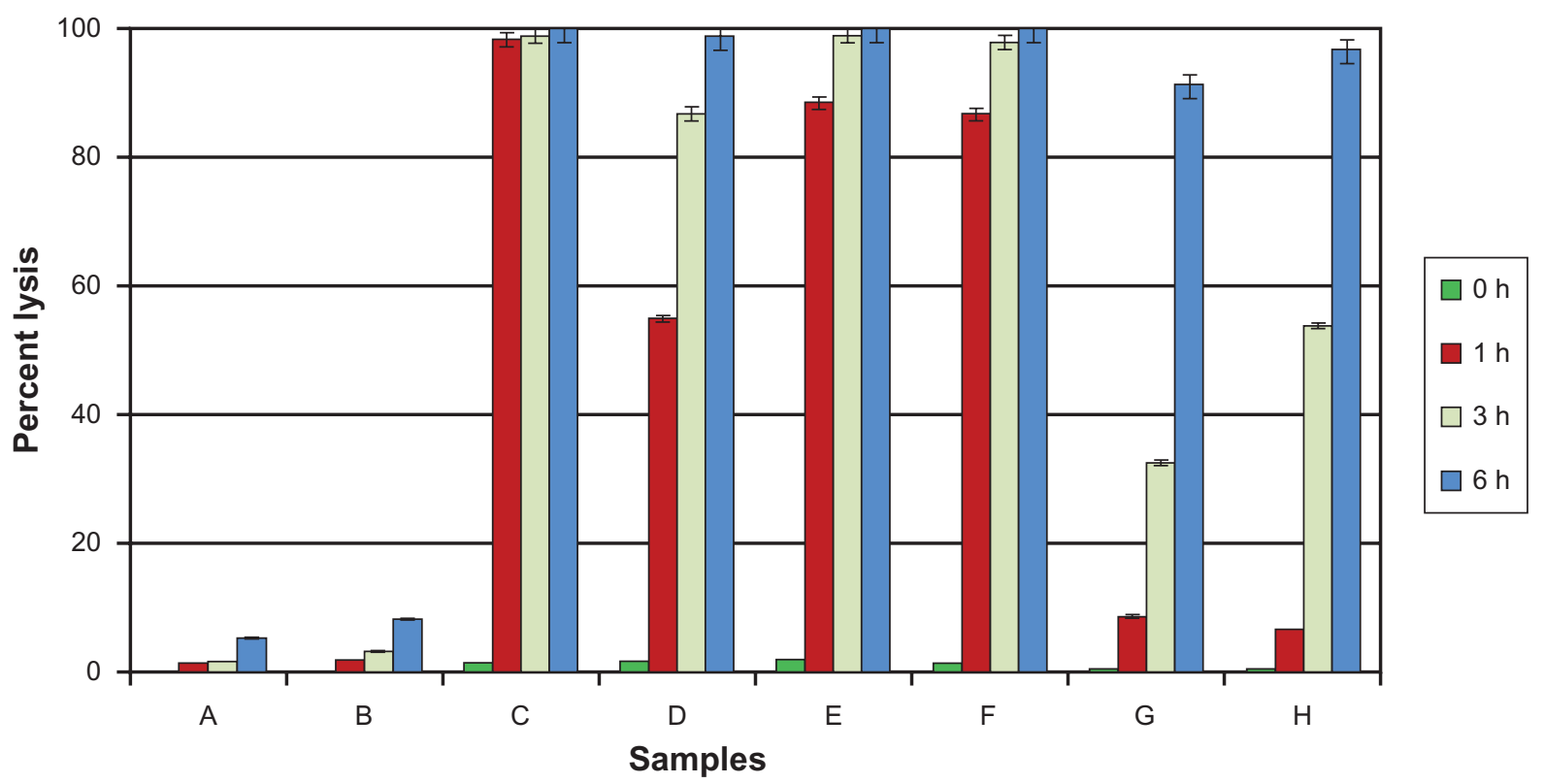

Figure 6 Chart showing the time-dependent hemolytic activity of AmB and its complexes towards human red blood cells. Results are expressed as percentage of hemolysis. AmB $(2 \mid \mu M)$ /curcumin $(32 \mu M)$ was used throughout the experiment. The values reported are means \pm SD $(n=3)$. (Samples: A, DMSO (I\%); B, curcumin; C, AmB; $\mathrm{D}$, AmB-curcumin mixture; E, AmB-BSA complex; F, AmB-HSA complex; G, AmB-BSA-curcumin complex; H, AmB-HSA-curcumin complex).

Abbreviations: AmB, amphotericin B; BSA, bovine serum albumin; DMSO, dimethyl sulfoxide; HSA, human serum albumin.

When both the components are protein bound, the process of lysis is delayed even more (Figure 6, samples G and H). Significantly, however, this reduction of hemolysis was achieved without compromising the antifungal activity of the ternary complex and was seen to be equivalent to that of albumin-AmB complex and the drug alone (Table 1).

\section{Conclusion}

There are several reports on the interaction of $\mathrm{AmB}$ with albumin, and curcumin with albumin. These studies have focused more on understanding the nature of interaction between albumin and the ligand. Even though there are a few reports on the amelioration of toxic effects of certain drugs by curcumin, the use of the drug alone with curcumin in a protein-complexed form and its beneficial biological effects have not been reported.

The protein-bound $\mathrm{AmB}$ shows antifungal and erythrocyte membrane-damaging activities. This indicates that the translocation of the antifungal agent from the protein to target membrane occurs readily. Although two distinct sites were noted for AmB and curcumin in the in silico study, there is no unequivocal evidence to show that the albumin-drug complex used in the present study is loaded with both ligands on all the protein molecules. It is quite likely that the complex preparation consists of a mixture of albumin molecules, some carrying both ligands, some with one ligand, and some free protein molecules.

The presence of curcumin in proximity to $\mathrm{AmB}$ may not have much significance in so far as the biological effect is concerned. What is perhaps important is the availability of curcumin at the site of action of AmB. It may be relevant to mention here that Ali et al reported the amelioration of the nephrotoxic effect of gentamicin by curcumin in rats. ${ }^{18}$ In this case, gentamicin was administered intramuscularly, while curcumin was given orally.

There is also a report of enhancement of antifungal activity of AmB or fluconazole towards Candida species by curcumin. No synergistic effect was observed at the concentration of curcumin used by us.

Cucumin has been shown to have protective effect on primaquine-induced oxidative damage. ${ }^{19} \mathrm{AmB}$ is thought to damage the mammalian cell membrane by formation of channels traversing membranes, and this requires selfassociated molecules. ${ }^{2}$ Whatever the mechanism of the damage process, we have also observed that with free $\mathrm{AmB}$ and curcumin together, the red cell lysis process is delayed. But in the protein complexed form, this protective effect is much more pronounced.

Kala-azar (visceral leishmaniasis) disease caused by protozoal parasite Leishmania donovani responds to treatment with AmB. In the light of the finding reported here, it may be worthwhile to investigate the therapeutic use of a combination of $\mathrm{AmB}$ and curcumin in the protein bound form.

\section{Disclosure}

The authors report no conflicts of interest in this work. 


\section{References}

1. Barrett JP, Vardulaki KA, Conlon C, et al. A systematic review of the antifungal effectiveness and tolerability of amphotericin B formulations. Clin Ther. 2003;25(5):1295-1320.

2. Brajtburg J, Bolard J. Carrier effects on biological activity of amphotericin B. Clin Microbiol Rev. 1996;9(4):512-531.

3. Butler WT, Cotlove E. Increased permeability of human erythrocytes induced by amphotericin B. Infect Dis. 1971;123:341-350.

4. Romanini D, Müller G, Pico G. Use of amphotericin B as optical probe to study conformational changes and thermodynamic stability in human serum albumin. J Protein Chem. 2002;21(8):505-514.

5. Chaubal MV. Human serum albumin as a pharmaceutical excipient. Drug Delivery Tech. 2005;5(8).

6. Mendez CM, McClain CJ, Marsano LS. Albumin therapy in clinical practice. Nutr Clin Prac. 2005;20(3):314-320.

7. Bourassa P, Kanakis CD, Tarantilis P, Pollissiou MG, Tajmir-Riah HA. Resveratrol, genistein, and curcumin bind bovine serum albumin. JPhys Chem B. 2010;114(9):3348-3354.

8. Morris GM, Goodsell DS, Halliday RS, et al. Automated docking using a Lamarckian genetic algorithm and empirical binding free energy function. J Comput Chem. 1998;19:1639-1662.

9. Cybuiska BJ, Mazerski E, Borowski, Gary-Bobo GM. Haemolytic activity of aromatic heptaenes, a group of polyene antifungal antibiotics. Biochem Pharmacol. 1984;33:41-46.

10. Brajtburg J, Elberg S, Schwartz DR, et al. Involvement of oxidative damage in erythrocyte lysis induced by amphotericin B. Antimicrob Agents Chemother. 1985;27(2):172-176.

11. Aggarwal BB, Bhatt ID, Ichikawa H, et al. Curcumin: Biological and Medicinal Properties in Turmeric: The Genus Curcuma. Baton Rouge, FL: CRC Press; 2007:297-368.
12. Anand P, Kunnumakkara AB, Newman RA, Aggarwal BB. Bioavailability of curcumin: problems and promises. Mol Pharm. 2007;4(6):807-818.

13. Bisht S, Feldmann G, Soni S, Ravi R, Karikar C, Maitra A. Polymeric nanoparticles encapsulated curcumin ("nanocurcumin"): a novel strategy for human cancer therapy. J Nanobiotechnology. 2007;5:3.

14. Narayanan NK, Nargi D, Randolph C, Narayanan BA. Liposome encapsulation of curcumin and resveratrol in combination reduces prostate cancer incidence in P TEN knockout mice. Int J Cancer. 2009; 125(1):1-8.

15. Tsao S, Yin M. Enhanced inhibitory effect from interaction of curcumin with amphotericin B or fluconazole against Candida species. J Food Drug Anal. 2000;8(3):208-212.

16. Barik A, Priyadarsini KI, Hari Mohan. Photophysical studies on binding of curcumin to bovine serum albumin. Photochem Photobiol. 2003; 77(6):597-603.

17. Barik A, Mishra B, Kunwar A, Priyadarsini KI. Interaction of curcumin with human serum albumin: thermodynamic properties, fluorescence energy transfer and denaturation effects. Chem Phys Lett. 2007; 436(1-3):239-243.

18. Ali BH, Al-Wabel N, Mahmoud O, Mousa HM, Hashad M. Curcumin has a palliative action of gentamicin-induced nephrotoxicity in rats. Fundam Clin Pharmacol. 2005;19:473-477.

19. Tonnesen HH, Kristensen S, Grinberg LN. Studies on curcumin and curcuminoids. XXV. Inhibition of primaquine-induced lysis of human red blood cells by curcumin. Int J Pharm. 1994;110(2):161-167.

20. National Committee for Clinical Laboratory Standards. Reference Method for Broth Dilution Antifungal Susceptibility Testing of Yeasts. Approved Standard; Document M-27A. Wayne, PA: National Committee for Clinical Laboratory Standards; 1997.
Journal of Experimental Pharmacology

\section{Publish your work in this journal}

The Journal of Experimental Pharmacology is an international, peerreviewed, open access journal publishing original research, reports, reviews and commentaries on all areas of laboratory and experimental pharmacology. The manuscript management system is completely online and includes a very quick and fair peer-review system.

\section{Dovepress}

Visit http://www.dovepress.com/testimonials.php to read real quotes from published authors. 